\title{
Unsteady flow and heat transfer past an axisymmetric permeable shrinking sheet with radiation effect.
}

\begin{abstract}
In this paper, the problem of unsteady axisymmetric boundary layer flow and heat transfer induced by a permeable shrinking sheet in the presence of radiation effect is studied. The transformed boundary layer equations are solved numerically by an implicit finite-difference scheme known as the Keller-box method. The influence of radiation, unsteadiness and mass suction parameters on the reduced skin friction coefficient $f^{\prime \prime}(0)$ and the heat transfer coefficient $-\theta^{\prime}(0)$, as well as the velocity and temperature profiles are presented and discussed in detail. It is found that dual solutions exist and suction parameter delays the separation of boundary layer.
\end{abstract}

Keyword: Unsteady flow; Heat transfer; Radiation effect; Boundary layer; Permeable shrinking sheet; Numerical solution. 